\title{
Feedback Control of Glucocorticoid Production is Established during Fetal Development
}

\author{
Holger M. Reichardt and Günther Schütz \\ Division Molecular Biology of the Cell I, German Cancer Research \\ Center, Heidelberg, Germany
}

\begin{abstract}
Background: Glucocorticoids are involved in the regulation of metabolic, immunological, and developmental processes. Their synthesis is tightly controlled by feedback regulation through the hypothalamus-pituitaryadrenal (HPA) axis, allowing the organism to respond to stress in an adequate manner and to adapt to new situations. Disturbance of these regulatory mechanisms leads to major human diseases. By generating mice with a targeted mutation in the glucocorticoid receptor (GR) locus, it was possible to analyze the mechanism by which glucocorticoids control the HPA axis, under conditions where at least part of the feedback control was absent early in development.

Materials and Methods: RNase-protection and in situ hybridization assays were used to compare messenger RNA (mRNA) levels of genes involved in the control of the HPA axis in both GR-mutant and wild-type animals.
\end{abstract}

Results: Negative feedback regulation of the HPA axis by glucocorticoids, which is established around Day E16.5 of embryonic development in wild-type mice, does not occur in GR-mutants, resulting in an increased expression of proopiomelanocortin mRNA in the anterior lobe of the pituitary and of corticotropin-releasing hormone mRNA in the paraventricular nucleus of the hypothalamus. However, the expression of both arginine vasopressin and mineralocorticoid receptor in the brain is not affected. In the neurointermediate lobe of the pituitary, expression of the proopiomelanocortin gene was inversely regulated, compared with its expression in the anterior lobe.

Conclusions: GR-dependent regulation of the HPA axis is established during fetal development, suggesting that maternal factors have an important role in influencing the HPA axis of the adult offspring.

\section{INTRODUCTION}

Glucocorticoids are synthesized in the adrenal gland and are involved in regulating metabolic, immunological, and developmental processes. Most of their physiological effects are mediated by the glucocorticoid receptor (GR), a liganddependent transcription factor which belongs to the steroid receptor superfamily (1). Although glucocorticoids bind to the mineralocorticoid receptor (MR) with higher affinity than to the GR, most of their effects are mediated via the GR, due to its wider expression and stronger transactivation potential. The generation of GR-deficient

Address correspondence and reprint requests to: Günther Schütz, Division Molecular Biology of the Cell I, German Cancer Research Center, Im Neuenheimer Feld 280, D-69120 Heidelberg, Germany. mice, by gene targeting in embryonic stem cells (2), made it possible to address in detail the role of the GR in controlling the hypothalamus-pituitary-adrenal (HPA) axis.

The HPA axis, which is activated during stress response, has an adaptive and life-sustaining function. The induced release of glucocorticoids by the adrenal cortex is the final step of a neuroendocrine cascade that begins in the central nervous system (CNS) and converges on the neuroendocrine cells in the paraventricular nucleus (PVN) of the hypothalamus. Hypophysiotropic factors then drive transcription of the proopiomelanocortin (POMC) gene and secretion of its major product, the adrenocorticotrope hormone (ACTH), from the anterior lobe of the pituitary (AL) and, thereby, stimulate glucocorticoid release. The level of glucocorticoids is tightly controlled by feedback inhibition involv- 
ing multiple components, including the pituitary, the hypothalamus, and the hippocampus. When corticosteroids are experimentally removed by adrenalectomy, a marked increase in transcription of the POMC gene in the AL and in the synthesis and secretion of ACTH are observed (3). In contrast, it is not clear whether POMC gene expression in the neurointermediate lobe (NIL) is also under control of glucocorticoids (4). In addition to its effects on the pituitary, adrenalectomy also removes the feedback signal for the hypothalamic peptides, corticotropin-releasing hormone $(\mathrm{CRH})$ and arginine vasopressin (AVP), leading to an increased biosynthesis of both factors $(5,6)$. Acute stress is a potent activator of the HPA axis; interestingly, the HPA axis retains the ability to respond to repeated stress despite high levels of corticosterone in the blood. This maintenance of sensitivity to stress is thought to be mediated by switching from a steroid-dependent to a steroid-independent pathway during the modulation of the HPA axis, possibly by changing from $\mathrm{CRH}$ to AVP as the main ACTH secretagogue $(7,8)$.

During development glucocorticoid synthesis starts at around Day E14 and peaks at birth (9), whereas expression of the GR gene is already observed at Day E10.5 (T. Cole, unpublished observation). In newborn mice carrying a targeted mutation in the GR locus, components of the HPA axis were found to be affected. The serum levels of corticosterone and ACTH, as well as the expression level of several stereoidogenic enzymes, were dramatically increased and the adrenal cortex showed a marked hypertrophy which was already evident at Day E15.5 (2). As the GR is a key player in the feedback regulation of corticosterone synthesis and secretion via the HPA axis, further studies were necessary to elucidate in detail the mechanism by which it controls these two pathways.

The GR-mutant mice represent a valuable tool to analyze the regulation of the HPA axis under conditions in which at least part of the feedback control is absent early in development and, therefore, to elucidate which regulatory mechanisms are active during embryogenesis. During our studies, we found that POMC and $\mathrm{CRH}$ expression are under negative feedback control as early as Day E16.5, whereas this same mechanism of regulation was not demonstrated for AVP and MR expression.

\section{MATERIALS AND METHODS}

\section{GR-Mutant Animals and Genotyping}

Mice with a targeted mutation in the glucocorticoid receptor gene (2) were housed under lightcontrolled conditions. GR-mutant embryos were obtained from heterozygous intercrosses and recovered by cesarian section. By convention, the day of the vaginal plug was counted as Day E0.5. Only littermates were compared in each experiment. Adult GR-mutant mice were matched and sacrificed at about 6 month of age.

For genotyping, a piece of the tail or the embryo was removed and digested with proteinase $\mathrm{K}$ overnight at $56^{\circ} \mathrm{C}$. After DNA preparation the genotype was determined either by Southern blot (2) or by polymerase chain reaction (PCR) analysis. The sequence of the primers used in the PCR reaction are the following: PG/3, 5'-AGAATCCTTAGCTCCCCCTGG-3' (exon 2-specific, upstream of the insertion site); PG/4, 5'CTGCTGCTTGGAATCTGCCTG-3' (exon 2-specific, downstream of the insertion site); PGK1, 5'TGCCTGCTCTTTACTGAAGGCT-3' (specific to the PGK-terminator used in the neo-selection cassette). PCR was performed using an established protocol with an annealing temperature of $64^{\circ} \mathrm{C}$ and 35 cycles. Amplified fragments were separated on a $1.5 \%$ agarose gel and visualized by ethidium bromide staining. A 170-bp band represented the wild-type allele and a 450-bp band the mutant one.

\section{RNA Isolation and RNase Protection Analysis}

Adult or embryonic heads were dissected and total RNA was isolated after homogenization in guanidinium thiocyanate (10). Quantification and quality control was done by photometry at $260 \mathrm{~nm} / 280 \mathrm{~nm}$ and by ethidium bromide staining of rRNAs after electrophoretic separation in denaturing agarose gels. RNase protection analysis was performed as previously described (11) using $\left[{ }^{32} \mathrm{P}\right]-\alpha \mathrm{UTP}-$ labeled antisense RNA probes. A 338-bp subclone of the mouse TBP (TATAbinding protein) gene (12) and a 110-bp subclone of mouse COX (cytochrome oxidase 1) gene were used as internal controls to normalize the amount of protected fragments. The antisense probes were hybridized overnight against appropriate amounts of total RNA at $54^{\circ} \mathrm{C}$ in $80 \%$ formamide. Excess probes were removed by digestion with RNases $\mathrm{A}$ and $\mathrm{Tl}$ and the protected fragments were analyzed on denaturing 
$5 \%$ or $6 \%$ polyacrylamide gels. Gels were exposed to an autoradiographic film and bands were subsequently quantified using the PhosphorImager system (Molecular Dynamics, Sunnyvale, CA).

For the synthesis of the 440-nt POMC antisense probe, a plasmid containing the whole coding region (kindly provided by J. Drouin) was linearized with NcoI and transcribed using T7polymerase. For AVP, a plasmid containing the 230-bp SmaI/PstI-fragment of the rat cDNA (kindly provided by E. Mohr) was used to generate an antisense probe. The protected 110-nt fragment in mouse RNA preparations was shown to be reproducible and specific (data not shown). The MR probe was synthesized by linearizing the plasmid pmMRex20.7P (kindly provided by $\mathrm{S}$. Berger) with $N c o$ I followed by transcription with T3-polymerase, yielding a 580-bp exon 2-specific probe.

\section{In Situ Hybridization}

Embryos, embryonic heads, or adult brains were fixed in $4 \%$ paraformaldehyde in phosphatebuffered saline (PBS; pH 7.2) overnight, dehydrated through an ethanol series, cleared in toluene, and embedded in paraffin. Six-micrometer sections from each stage and tissue were sealed on TESPA-treated slides. In situ prehybridizations and hybridizations were carried out as described (13). Hybridizations were done overnight at $58^{\circ} \mathrm{C}$ in $50 \%$ formamide using $\left[{ }^{35} \mathrm{~S}\right]-\alpha \mathrm{UTP}-$ labeled antisense RNA probes at a concentration of $60 \mathrm{ng} / \mathrm{ml}$. The first posthybridization wash was performed at $62^{\circ} \mathrm{C}$, the second at $68^{\circ} \mathrm{C}$, and both in $50 \%$ formamide. The final wash in $0.1 \times$ SSC at $55^{\circ} \mathrm{C}$ was done before dehydration of the sections. Slides were then dipped in Kodak NTB2 emulsion diluted $1: 1$ with water, exposed at $4^{\circ} \mathrm{C}$ for 2 to 17 days, and developed using Kodak D19 developing solution and Kodafix at $15^{\circ} \mathrm{C}$ for 4 min. Sections were stained using eosin and hematoxylin and visualized using a Zeiss Axiophot microscope.

The POMC plasmid (see above) was used to synthesize a 923-nt antisense probe covering the whole coding sequence of the mouse POMC gene. The CRH probe was made from a plasmid containing a 578-bp PstI-fragment from exon 2 of the mouse gene (kindly provided by $\mathrm{A}$. Seasholtz). For AVP, the same rat cDNA clone described above was used to generate a 230-nt antisense probe. The specifity of all three probes was confirmed by the region-specific expression pattern.

\section{RESULTS}

\section{POMC Gene Transcription in the Pituitary Becomes Responsive to GR-Mediated Regulation between Days E14.5 and E16.5 of Development}

When adrenals are experimentally removed, the decrease in corticosterone levels in the blood leads to an increase in the transcription of the POMC gene in the anterior lobe of the pituitary, and an increased synthesis and secretion of ACTH. Similarly, in the absence of functional GR, feedback control via the HPA axis is impaired and a dramatic increase in ACTH levels is found in homozygous newborn mice (2). Based on these observations, we wanted to investigate whether this increase in ACTH levels in the GRdeficient mice could be attributed to an up-regulation of POMC gene transcription and, also, at which point during development it occurs.

POMC mRNA levels were analyzed by in situ hybridization at stages E14.5, E16.5, and E18.5, as well as in adult pituitaries. At E14.5, a clear signal was detected in the AL but not in the NIL. A qualitative comparison of POMC mRNA level in the $\mathrm{AL}$ of wild-type and mutant animals showed no difference (Fig. 1). However, analysis of E16.5 embryos revealed a dramatic difference. Here, the expression level of the POMC gene was found to be much higher in mutants than in wild-type animals (Fig. 1). It thus appears that suppression of POMC transcription by the GR becomes effective starting at around Day E16.5 of development. A surprising observation was made with regard to POMC expression in the NIL: a strong reduction in POMC mRNA levels was observed in mutant animals compared with wild-type (Fig. 1). This shows that expression of the POMC gene is regulated in an opposite manner in the two lobes of the pituitary.

When E18.5 and adult pituitaries were analyzed, the overall level of POMC expression was slightly increased, but the observed qualitative difference in expression between wild-type and mutant animals remained the same. This difference was more pronounced in E18.5 fetuses than in adults.

In order to estimate the magnitude of the changes in POMC mRNA levels, pituitaries of adult mice were dissected, AL and NIL/PL were separated, and RNA was prepared. Pooled fractions were analyzed for POMC mRNA levels by RNase protection assays and quantified as described in Materials and Methods. In the AL, POMC expression increased by $72 \%$ in GR-mu- 

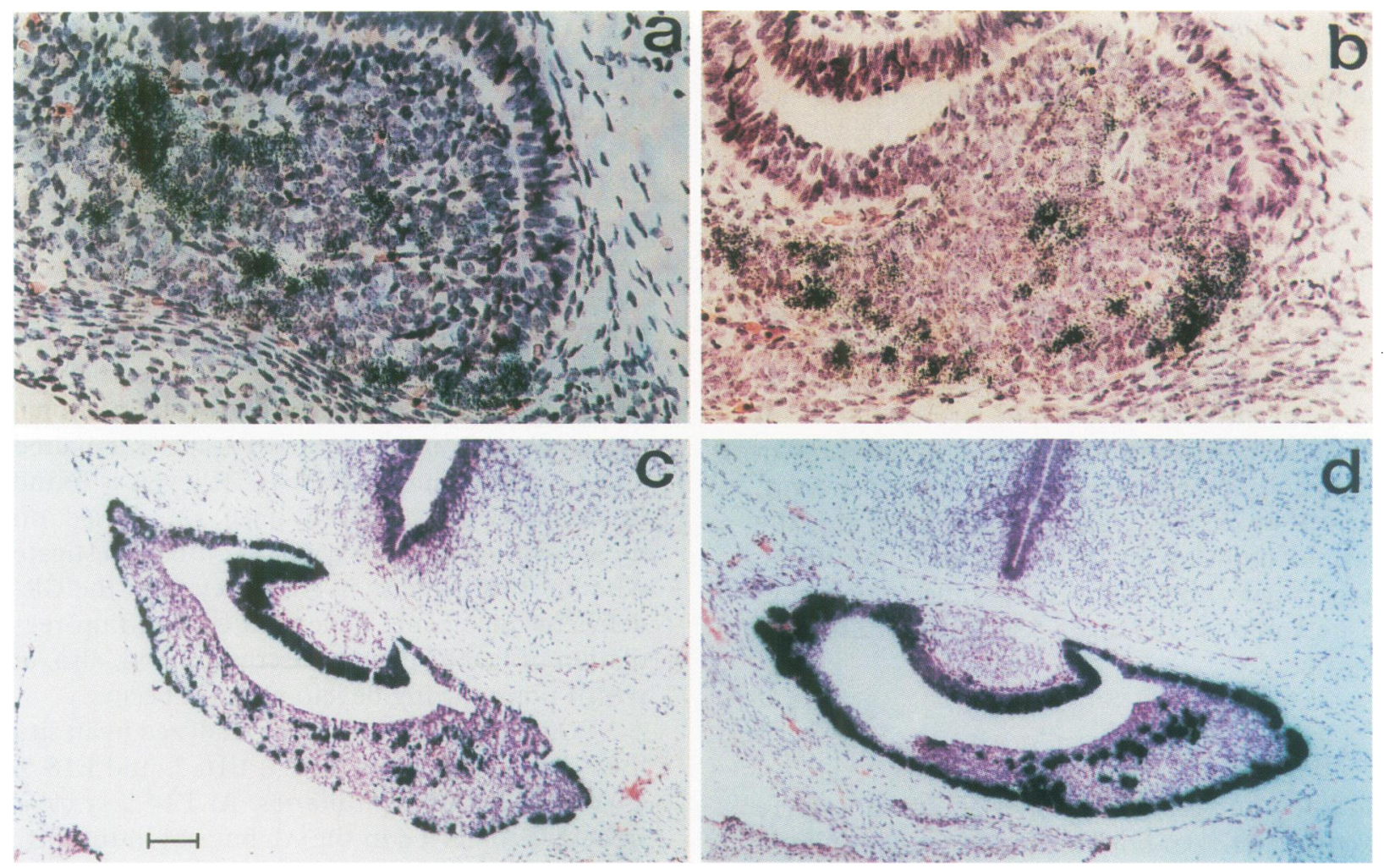

FIG. 1. Expression of POMC mRNA in fetal pituitaries

Sagital sections through the head of E14.5 embryos ( $a$ and $b$ ) and coronal sections through the head of E16.5 embryos ( $c$ and d). Expression of POMC in wild-type embryos ( $a$ and c) and homozygous mutant embryos (b and d) is compared. Bar $=25 \mu \mathrm{m}(\mathrm{a}$ and $\mathrm{b})$ and $100 \mu \mathrm{m}$ (c and d).

tants compared with wild-type animals, whereas in the NIL it decreased to $30 \%$ of that in wildtype animals (Fig. 2). The absolute expression level of POMC is much higher in the NIL (approximately 14 -fold, indicated by a different scale in the figure) due to the fact that it represents a nearly pure population of POMC-expressing melanotrophes, whereas the corticotrophes in the AL make up only about $10 \%$ of the hormone-producing cells.

By comparing the in situ hybridization data obtained from embryonic and adult pituitaries, it can be estimated that the increase of POMC expression in the embryonic AL is much higher than the one observed in adults, whereas the differences observed in the NIL seem to be roughly the same at all stages of development after E16.5 (data not shown).

\section{CRH Expression Is Increased in GR-Mutant Mice Starting around E16.5 of Development}

It has been known for some time that an additional level of the feedback regulation of glu- cocorticoid production exists in the hypothalamus. In particular, $\mathrm{CRH}$, which is the main secretagogue for ACTH, is thought to be under control of the GR. Therefore, we analyzed mRNA levels of CRH in different regions of the brain of wild-type and mutant animals.

Fetal and adult brains were analyzed by in situ hybridization. The first time point used was E16.5; a strong and selective signal in the PVN of the hypothalamus was obtained in wild-type animals. When mutants of the same stage were analyzed, a slight but reproducible increase in CRH transcription in the PVN was observed (Fig. 3). The magnitude of this effect was estimated to correspond to about a $50 \%$ increase. In adult brains, CRH mRNA expression was increased 2 -fold in mutant animals. To test if the observed effect was specific, mRNA levels in other regions of the adult brain known to express CRH (14) were investigated. No differences in expression between mutant and wild-type animals were found in the medial geniculate nucleus, the Barringtons nucleus, or the cortex (data not shown). 

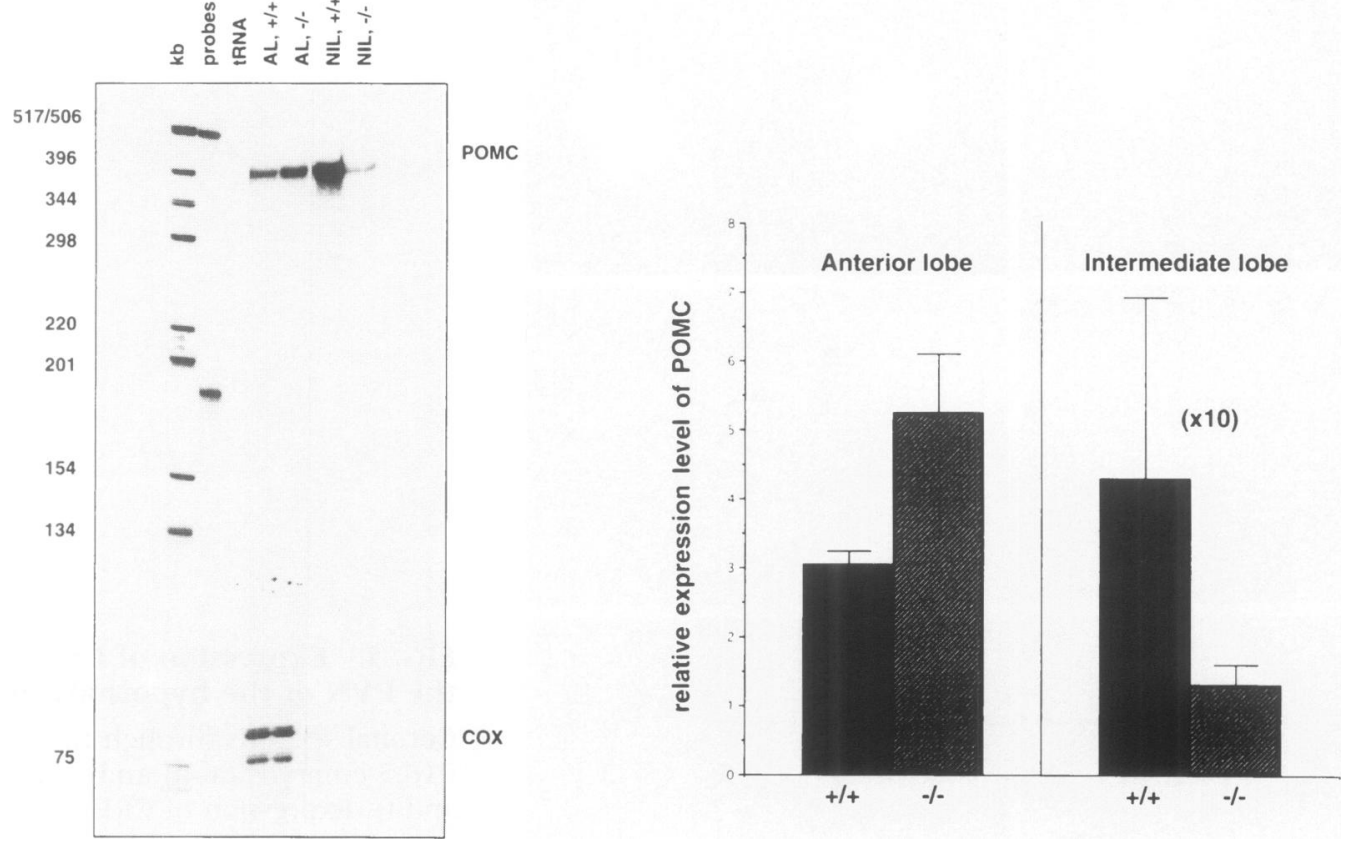

FIG. 2. Expression of POMC mRNA in the AL and NIL of adult pituitaries

(a) One microgram (AL) and $0.2 \mu \mathrm{g}$ (NIL) of RNA from pooled pituitary lobes were analyzed by RNase protection as described in Materials and Methods. Protected fragments for POMC and COX (cytochrome oxidase 1), as well as molecular size markers, are indicated. (b) Bands were quantified and normalized to loaded amount of RNA using the expression of COX. Data represent mean \pm SEM (bars) of two independent experiments expressed as the ratio of POMC/COX expression levels. Note the different scales for AL and NIL.

\section{AVP Expression in the Hypothalamus Is Not Altered in GR-Mutant Mice}

The onset of expression of AVP occurs rather late during murine development compared with that of CRH: in the supraoptical nucleus (SON), AVP mRNA is first detected around E15; in the PVN, expression begins at E18; and, in the suprachiasmatic nucleus (SCN), even later $(15,16)$.

In an attempt to investigate AVP expression in GR-mutant mice, total RNA was isolated from brains of newborn mice and analyzed by RNAase protection. No changes in the mRNA levels were found in comparison with wild-type animals (data not shown). Since subtle changes could not be excluded, a more refined analysis of AVP expression in the hypothalamus of adult mice was conducted. RNase protection analyses of hypothalamic RNA preparations did not show any changes in AVP mRNA levels in GR-mutants compared with wild-type animals (Fig. 4). To address the question of whether changes in individual nuclei of the hypothalamus occur, adult brains were analyzed by in situ hybridization. Changes in AVP expression were not observed in either the PVN, the SON (Fig. 5), or the SCN (data not shown). Although no clear distinction between parvocellular and magnocellular neurons could be made, a major difference in AVP expression in these neurons is unlikely according to the data presented. It thus seems reasonable to conclude that AVP is not a major target for transcriptional regulation by the GR.

\section{MR Expression in the Hippocampus Is Unaltered in GR-Mutant Mice}

The integration of learning, mood, behavioral patterns, and the control of the HPA axis is thought to occur at the level of the hippocampus. Although it is not clear which neuronal projections are involved (17), there is strong evidence that the hippocampus controls HPA axis activity and is itself a target for glucocorticoid negative feedback. Both the GR and the MR are expressed in the hippocampus at relatively high levels, and, since both proteins are able to bind glucocorticoids (18), it was tempting to speculate that the 

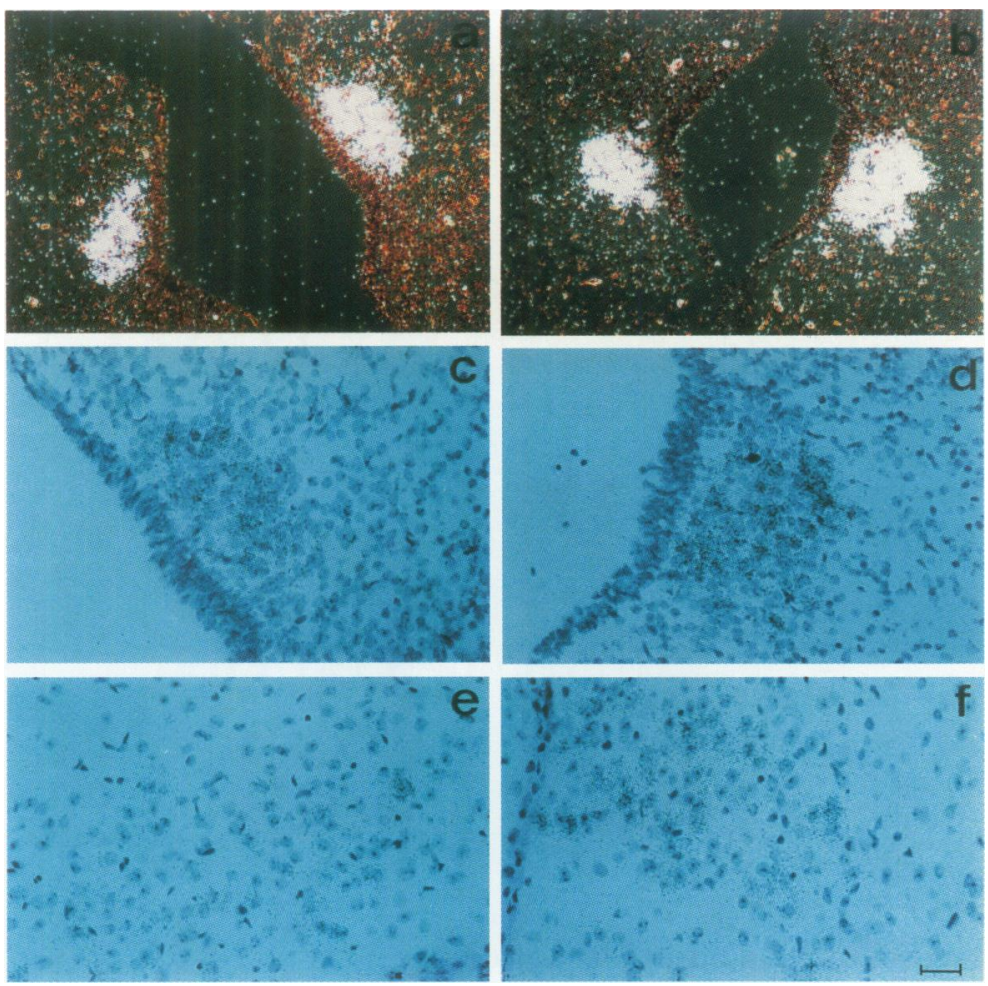

FIG. 3. Expression of CRH mRNA in the PVN of the hypothalamus

Coronal sections through the head of E16.5 embryos (a-d) and adult brains (e and $f$ ). Expression of CRH in wild-type mice (a, c, and e) and homozygous mutant mice (b, d, and f) is compared. Bar $=50 \mu \mathrm{m}(\mathrm{a}$ and $\mathrm{b})$ and $25 \mu \mathrm{m}(\mathrm{c}-\mathrm{f})$.

lack of functional GR could influence expression of the MR.

Total RNA obtained from hippocampi of adult wild-type and mutant mice was prepared and analyzed by RNAase protection. Surprisingly, no change in the overall expression level of MR could be demonstrated (Fig. 6). Although, regional differences in expression within the hippocampus would not be detected by the methodology employed, it seems unlikely that an increase in one area compensated for a decrease in another. Therefore, it can be concluded that MR expression is not directly controlled by the GR.

\section{DISCUSSION}

\section{POMC Expression in the AL of the Pituitary}

The availability of mice with a targeted mutation in the GR locus offers the exciting possibility of characterizing the control of glucocorticoid synthesis via the HPA axis, the establishment of the HPA axis during fetal development, and the contributions of various components in the feedback control circuit. Hence, we have analyzed the regulation of the HPA axis in GR-mutant mice during development and in adulthood. The results

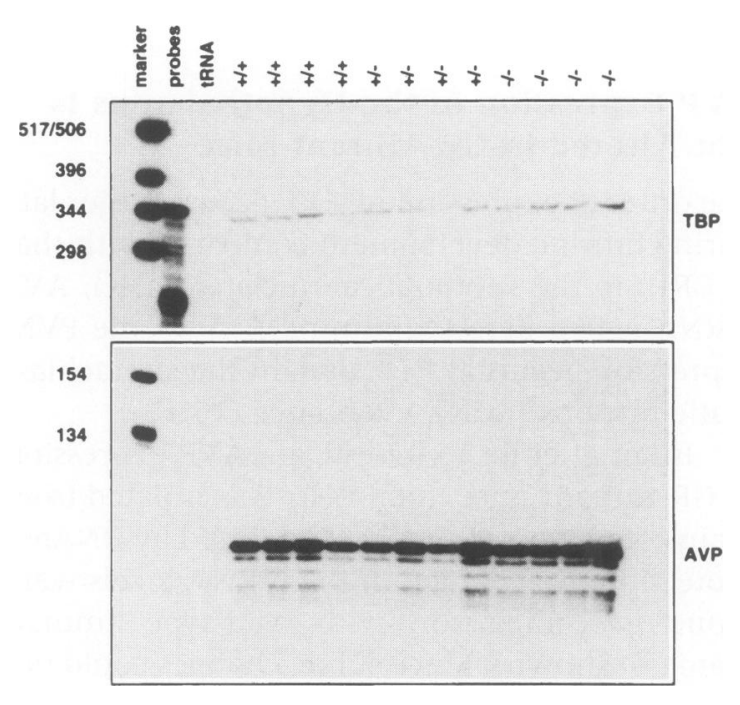

FIG. 4. Expression of AVP-mRNA in adult hypothalamus

Two micrograms of RNA from hypothalamus were analyzed by RNAase protection as described in Materials and Methods. Protected fragments for TBP and AVP, as well as molecular size markers, are indicated. Bands were quantified and normalized to loaded amount of RNA using the expression of TBP. No difference between the three genotypes was found. 

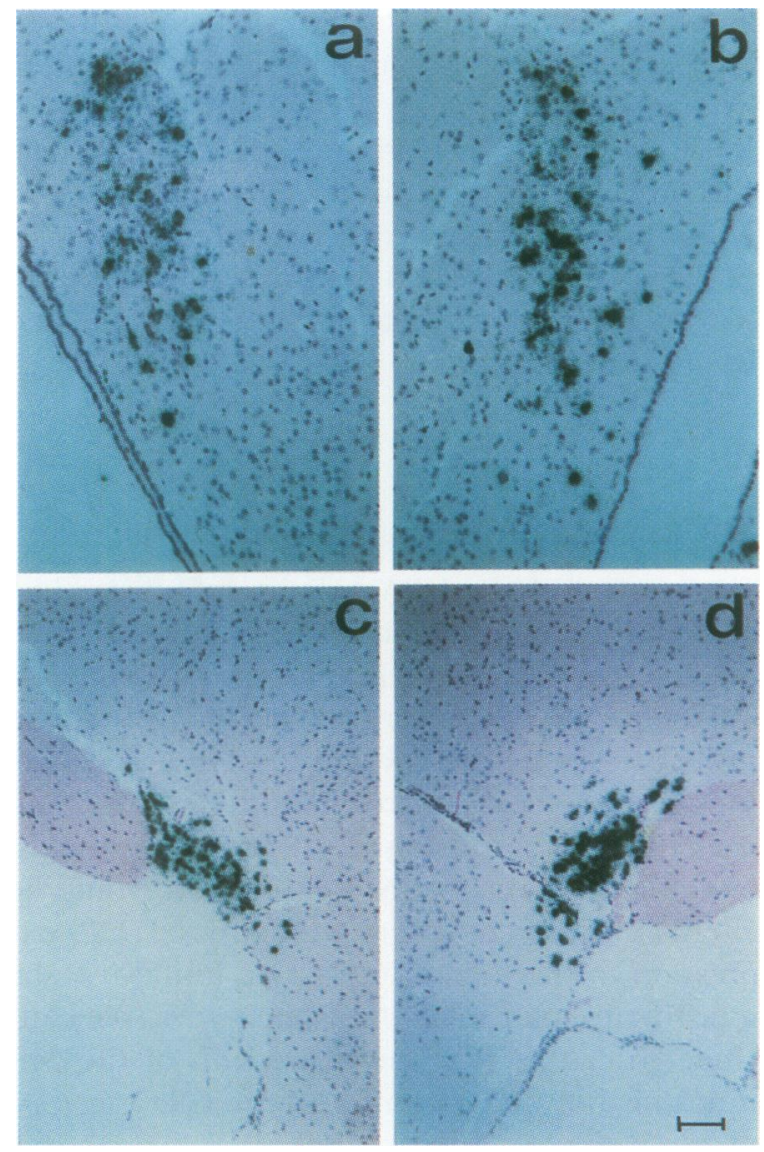

FIG. 5. Expression of AVP mRNA in the PVN ( $a$ and $b$ ) and the SON (c and $d$ ) of the hypothalamus in adult mice

Expression in wild-type (a and c) and mutant mice (b and d) was compared. Bars $=50 \mu \mathrm{m}$ ( $\mathrm{a}$ and $\mathrm{b}$ ) and $100 \mu \mathrm{m}$ (c and d).

presented here demonstrate that the expression of genes involved in the control of the HPA axis is under the influence of the GR as early as Day E16.5 of development. When fetal pituitaries were analyzed for POMC expression, no change was found in the anterior lobe at E14.5, whereas POMC expression was strongly increased by Day E16.5 and later time points. This observation is consistent with the identification of a negative glucocorticoid-responsive element (GRE) in the control region of the mouse POMC gene (19). So far, only in vitro experiments had shown that fetal rat pituitaries are capable of ACTH release, which could be attenuated by corticosterone $(20,21)$. However, there had been no evidence for negative feedback control occurring at the transcriptional level during development, nor any indication as to when this feedback would become effective. The early onset of the regula-

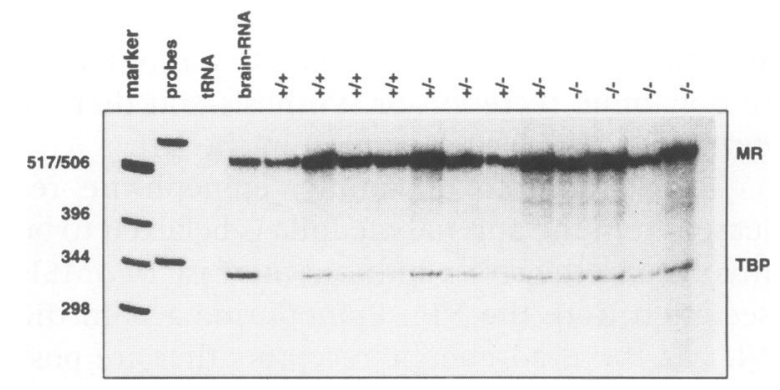

FIG. 6. Expression of MR mRNA in adult hippocampus

Three micrograms of RNA from hippocampus and 10 $\mu \mathrm{g}$ from whole brain were analyzed by RNAase protection as described in Materials and Methods. Protected fragments for TBP and MR, as well as molecular size markers, are indicated. Bands were quantified and normalized to loaded amount of RNA using the expression of TBP. No difference between the three genotypes was found.

tion of POMC transcription by the GR suggests that important components of the HPA axis are already operative during fetal development.

\section{POMC Expression in the NIL}

An unexpected observation was that POMC expression was significantly decreased in the intermediate lobe of the pituitary. In adult GR-deficient animals, POMC mRNA in the NIL was measured to be $30 \%$ of that in wild-type. This result was surprising, because most investigators have not so far reported an effect of glucocorticoids on intermediate lobe POMC expression (3), except in one case, where long-term treatment with dexamethasone was found to increase the transcription level of POMC in the NIL (22). Since GR is not detectable in the NIL under physiological conditions, a direct effect in mutants is highly unlikely. The main regulatory mechanism to control POMC expression in melanotrophe cells of the NIL is the dopaminergic system. In several reports it has been shown that dopamine inhibits POMC transcription and $\alpha \mathrm{MSH}$ secretion. Both in adults and during development $(23,24)$. This action is mediated via D2 dopamine receptors, which are negatively coupled to adenylate cyclase. Dopaminergic neurons found in the NIL suggest that POMC synthesis and secretion are controlled by neuronal axons originating in the hypothalamus. In addition, it was shown that stress increases $\alpha \mathrm{MSH}$ secretion from the NIL and decreases dopamine levels (25). A pos- 
sible explanation would be that the high corticosterone levels during stress lead to an inhibition of dopamine secretion or synthesis and thereby derepress POMC transcription in the NIL.

In addition to dopamine, epinephrine released from the adrenal medulla is believed to be involved in the stress-induced increase of $\alpha \mathrm{MSH}$ secretion from the NIL. Epinephrine acts in the NIL via the $\beta$-adrenergic receptors that are positively coupled to adenylate cyclase, leading to a synergistic effect in the inhibition of dopamine secretion. In GR-mutant mice, epinephrine is dramatically reduced in the blood (2); also, corticosterone cannot inhibit the dopaminergic system via the GR. These combined effects could converge intracellularly by reducing the cAMP levels in melanotrophe cells and thereby inhibit POMC transcription in the NIL, possibly via the transcription factor CREB. The idea that the decrease in the POMC mRNA levels can be attributed, at least in part, to a deregulation of the dopaminergic system is supported by a report that the effects of pharmacological substances are smaller in males than in females (24), an observation that has also been made in adult GRmutants (unpublished data). Further studies will be necessary to analyze this mechanism in more detail.

\section{Hypothalamic Expression of CRH and AVP}

The feedback inhibition of the HPA axis is known to involve multiple components, including the hypothalamus. We found a marked difference in the expression of CRH and AVP, the main ACTH secretagogues in the hypothalamus. CRH transcription was already increased in the paraventricular nucleus of GR-deficient mice at Day E16.5; in contrast, there was no change in AVP expression in any area of the hypothalamus.

It has often been speculated that there are two different pathways by which POMC can be activated in the pituitary: a steroid-sensitive one and a steroid-insensitive one (7). If animals are acutely stressed, this leads to an up-regulation of CRH but not AVP expression. After repeated stress, however, the increase in AVP expression is much greater than that in $\mathrm{CRH}$, and this happens despite high levels of glucocorticoids in the blood (26). Obviously, CRH can be effectively suppressed by glucocorticoids, whereas AVP seems to be inducible under high corticosterone conditions. This suggests that a shift from CRH to AVP as the main ACTH secretagogue occurs in order to maintain the ability of the animal to respond to stress. This model is now supported by our observation with GR-mutant mice, which show a different regulation of both genes by the GR. In addition, the results are in agreement with those obtained with $\mathrm{CRH}$-deficient mice (27). These mice also do not show a difference in AVP expression, despite significantly decreased levels of corticosterone.

\section{MR Expression in the Hippocampus}

The control of the HPA axis by influences like behavior or immune challenge is thought to be mediated in part by the hippocampus. From various experiments, it can be concluded that the mRNA levels for the GR and the MR are subject to negative feedback regulation $(28,29)$, and, since the GR is missing in GR-mutant animals, one might expect that MR expression would be altered. However, when we analyzed MR expression in the hippocampus, we found it to be indistinguishable in all three genotypes. We conclude from this that the expression of the MR mRNA is not under control of the GR.

\section{Prenatal Control of the HPA Axis}

One of the most interesting findings was that both POMC and CRH gene expression are sensitive to GR-mediated regulation as early as Day E16.5 of development. This sheds new light on the phenomenon that prenatal experiences may influence the HPA axis in adulthood. Stress during pregnancy, as well as immunological challenge of the mother, was shown to be manifested in a change of HPA axis activity in the adult offspring (30). The observation that the expression level of key genes, like POMC and $\mathrm{CRH}$, is sensitive to changes in the corticosterone level in fetal development offers a possible explanation as to how stress and immunreactions could exert their influence on the HPA axis at this early stage. It is hypothesized that during the period when the HPA axis becomes established increased glucocorticoid levels could set a new nominal value to which the system gets adapted, resulting in an impaired HPA axis in adulthood. Clinically, one may postulate that prenatal stress could therefore increase the susceptibility to psychiatric disorders like depression and schizophrenia later in life. 


\section{Conclusion}

In conclusion, the present experiments show that the HPA axis is sensitive to GR-mediated regulation as early as Day E16.5 of development. POMC in the pituitary and CRH in the PVN of the hypothalamus represent genes that are under direct control of the GR; in contrast, a similar type of regulation could not be shown for hypothalamic AVP and hippocampal MR expression. The experiments presented here represent an initial approach to analyzing the basal regulation of the HPA axis in GR-mutant mice. It will be important in the future to determine how this altered system reacts to stressors and whether compensatory mechanisms or steroid-insensitive pathways allow the GR-mutant mice to respond to stress despite the high basal corticosterone levels.

\section{ACKNOWLEDGMENTS}

We would like to thank Dagmar Bock and Anja Schwäger for expert technical assistance and Werner Fleischer for oligonucleotide synthesis and photographical artwork. We are grateful to Drs. Klaus H. Kaestner, A. Paula Monaghan, and Wolfgang Schmid for helpful discussions, and to Dr. Klaus H. Kaestner for critical reading of the manuscript. This work was supported by the Deutsche Forschungsgemeinschaft through SFB 229, the Fonds der Chemischen Industrie, the BMFT project 0310681, and European Community Grant BI02-CT93-0319.

\section{REFERENCES}

1. Beato M, Herrlich P, Schütz G. (1995) Steroid hormone receptors: many actors in search of a plot. Cell 83: 851-857.

2. Cole TJ, Blendy JA, Monaghan AP, et al. (1995) Targeted disruption of the glucocorticoid receptor gene blocks adrenergic chromaffin cell development and severely retards lung maturation. Genes Dev. 9: 1608-1621.

3. Birnberg NC, Lissitzky JC, Hinman M, Herbert E. (1983) Glucocorticoids regulate proopiomelanocortin gene expression in vivo at the levels of transcription and secretion. Proc. Natl. Acad. Sci. U.S.A. 80: 6982-6986.

4. Autelitano DJ, Lundblad JR, Blum M, Roberts JL. (1989) Hormonal regulation of
POMC gene expression. Annu. Rev. Physiol. 51: 715-726.

5. Young III W. (1992) Regulation of gene expression in the hypothalamus: Hybridization histochemical studies. Ciba Found. Symp. 168: 127-138.

6. Plotsky PM, Sawchenko PE. (1987) Hypophysial-portal plasma levels, median eminence content, and immunohistochemical staining of corticotropin-releasing factor, arginine vasopressin, and oxytocin after pharmacological adrenalectomy. Endocrinology 120: 1361-1369.

7. Plotsky PM, Thrivikraman KV, Meaney MJ. (1993) Central and feedback regulation of hypothalamic corticotropin-releasing factor secretion. Ciba Found. Symp. 172: 59-84.

8. Sawchenko PE, Imaki T, Potter E, Kovacs K, Imaki J, Vale W. (1993) The functional neuroanatomy of corticotropin-releasing factor. Ciba Found. Symp. 172: 5-29.

9. Michelsohn AM, Anderson DJ. (1992) Changes in competence determine the timing of two sequential glucocorticoid effects on sympathoadrenal progenitors. Neuron 8: 589-604.

10. Chomczynski P, Sacchi N. (1987) Single-step method of RNA isolation by acid guanidinium thiocyanate-phenol-chloroform extraction. Anal. Biochem. 162: 156-159.

11. Kaestner KH, Ntambi JM, Kelly TJ, Lane MD. (1989) Differentiation-induced gene expression in 3T3-Ll preadipocytes. A second differentially expressed gene encoding stearoyl-CoA desaturase. J. Biol. Chem. 264: 14755-14761.

12. Tamura T, Sumita K, Fujino I, et al. (1991) Striking homology of the 'variable' N-terminal as well as the 'conserved core' domains of the mouse and human TATA-factors (TFIID). Nucleic Acids Res. 19: 3861-3865.

13. Wilkinson DG (ed). (1992) In Situ Hybridization. A Practical Approach. Oxford University Press, New York.

14. Keegan CE, Herman JP, Karolyi IJ, O'Shea KS, Camper SA, Seasholtz AF. (1994) Differential expression of corticotropin-releasing hormone in developing mouse embryos and adult brain. Endocrinology 134: 2547-2555.

15. Hyodo S, Yamada C, Takezawa T, Urano A. (1992) Expression of provasopressin gene during ontogeny in the hypothalamus of developing mice. Neuroscience 46: 241-250.

16. Ang HL, Carter DA, Murphy D. (1993) Neuron-specific expression and physiological 
regulation of bovine vasopressin transgenes

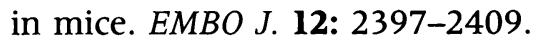

17. Cullinan WE, Herman JP, Watson SJ. (1993) Ventral subicular interaction with the hypothalamic paraventricular nucleus: Evidence for a relay in the bed nucleus of the stria terminalis. J. Comp. Neurol. 332: 1-20.

18. Joels M, de Kloet R. (1994) Mineralocorticoid and glucocorticoid receptors in the brain. Implications for ion permeability and transmitter systems. Prog. Neurobiol. 43: 1-36.

19. Drouin J, Sun YL, Chamberland M, et al. (1993) Novel glucocorticoid receptor complex with DNA element of the hormone-repressed POMC gene. EMBO J. 12: 145-156.

20. Dupouy JP, Chatelain A. (1984) In-vitro effects of corticosterone, synthetic ovine corticotrophin releasing factor and arginine vasopressin on the release of adrenocorticotrophin by fetal rat pituitary glands. J. Endocrinol. 101: 339-344.

21. Lugo IL, Pintar JE. (1996) Ontogeny of basal and regulated secretion from POMC cells of the developing anterior lobe of the rat pituitary gland. Dev. Biol. 1: 95-109.

22. Autelitano DJ, Clements JA, Nikolaidis I, Canny BJ, Funder JW. (1987) Concomitant dopaminergic and glucocorticoid control of pituitary proopiomelanocortin messenger ribonucleic acid and beta-endorphin levels. Endocrinology 121: 1689-1696.

23. Lugo DI, Pintar JE. (1996) Ontogeny of basal and regulated proopiomelanocortin-derived peptide secretion from fetal and neonatal pituitary intermediate lobe cells: Melanotrophs exhibit transient glucocorticoid responses during development. Dev. Biol. 173: $110-118$.
24. Chen CL, Dionne FT, Roberts JL. (1983) Regulation of the proopiomelanocortin mRNA levels in rat pituitary by dopaminergic compounds. Proc. Natl. Acad. Sci. U.S.A. 80: $2211-$ 2215.

25. Lindley SE, Lookingland $\mathrm{KJ}$, Moore $\mathrm{KE}$. (1990) Dopaminergic and beta-adrenergic receptor control of alpha-melanocyte-stimulating hormone secretion during stress. Neuroendocrinology 52: 46-51.

26. Makino S, Smith MA, Gold PW. (1995) Increased expression of corticotropin-releasing hormone and vasopressin messenger ribonucleic acid (mRNA) in the hypothalamic paraventricular nucleus during repeated stress: association with reduction in glucocorticoid receptor mRNA levels. Endocrinology 136: 3299-3309.

27. Muglia L, Jacobson L, Dikkes P, Majzoub JA. (1995) Corticotropin-releasing hormone deficiency reveals major fetal but not adult glucocorticoid need. Nature 373: 427-432.

28. Herman JP, Adams D, Prewitt C. (1995) Regulatory changes in neuroendocrine stressintegrative circuitry produced by a variable stress paradigm. Neuroendocrinology 61: 180190.

29. Holmes MC, Yau JL, French KL, Seckl JR. (1995) The effect of adrenalectomy on 5-hydroxytryptamine and corticosteroid receptor subtype messenger RNA expression in rat hippocampus. Neuroscience 64: 327-337.

30. Reul JM, Stec I, Wiegers GJ, et al. (1994) Prenatal immune challenge alters the hypothalamic-pituitary-adrenocortical axis in adult rats. J. Clin. Invest. 93: 2600-2607.

Contributed by B. W. O'Malley. Accepted on August 22, 1996. 